\title{
Deep Learning Forecasting in Cryptocurrency High-Frequency Trading
}

\author{
Salim Lahmiri ${ }^{1} \cdot$ Stelios Bekiros ${ }^{2,3}$ \\ Received: 28 October 2020 / Accepted: 15 January 2021/ Published online: 2 February 2021 \\ (c) The Author(s), under exclusive licence to Springer Science+Business Media, LLC part of Springer Nature 2021
}

\begin{abstract}
Background Like common stocks, Bitcoin price fluctuations are non-stationary and highly noisy. Due to attractiveness of Bitcoin in terms of returns and risk, Bitcoin price prediction is attracting a growing attention from both investors and researchers. Indeed, with the development of machine learning and especially deep learning, forecasting Bitcoin is receiving a particular interest.

Methods We implement and apply deep forward neural network (DFFNN) for the analysis and forecasting Bitcoin highfrequency price data. Importantly, we seek to investigate the effect of standard numerical training algorithms on the accuracy obtained by DFFNN; namely, the conjugate gradient with Powell-Beale restarts, the resilient algorithm, and LevenbergMarquardt algorithm. The DFFNN was applied to a big dataset composed of 65,535 samples.

Results In terms of root mean of squared errors (RMSEs), the simulation results show that the DFFNN trained with the Levenberg-Marquardt algorithm outperforms DFFNN trained with Powell-Beale restarts algorithm and DFFNN trained with resilient algorithm. In addition, the resilient algorithm is fast which suggests that it could be promising in online training and trading.
\end{abstract}

Conclusions The DFFNN trained with Levenberg-Marquardt algorithm is effective and easy to implement for Bitcoin highfrequency price data forecasting.

Keywords Bitcoin $\cdot$ High frequency $\cdot$ Deep learning $\cdot$ Forecasting

\section{Introduction}

According to the efficient-market hypothesis [1], stock prices follow a random walk, and it is impossible to forecast direction and magnitude changes. However, with the development of machine learning tools, various works have been employed in modeling and prediction stock prices [2] to generate better forecasts. Indeed, this task is a critical step in financial decision-making related to portfolio optimization,

Stelios Bekiros

stelios.bekiros@eui.eu

Salim Lahmiri

salim.lahmiri@concordia.ca

1 Department of Supply Chain and Business Technology Management, John Molson School of Business, Concordia University, Montreal, Canada

2 Department of Economics, European University Institute, Florence, Italy

3 IPAG Business School, Department of Finance and Information Systems, Paris, France risk evaluation, and trading. One of the hot topics in financial time series is to analyze and predict prices of cryptocurrencies by using machine learning. To cite the most recent works that focus on application in the relevant field, long-short-term memory and generalized neural networks were employed in [3]; neuro-fuzzy controller in [4];; combination of complete empirical ensemble mode decomposition, and support vector machine in [5]; artificial neural networks, support vector machines, and ensemble algorithms in [6]; decision trees in [7]; and support vector regressions, Gaussian Poisson regressions, regression trees, $k$-nearest neighbors algorithm, standard feed-forward neural networks, Bayesian regularization neural networks, and radial basis function neural networks [8].

Our goal is to implement and validate a deep feedforward neural network in the task of forecasting Bitcoin price at high-frequency sampling. Indeed, this is the first time DFFNN is designed to analyze hidden patterns in Bitcoin high-frequency price data for forecasting purpose. Additionally, we examine the effect of three standard training algorithms used to update DFFNN weights on 

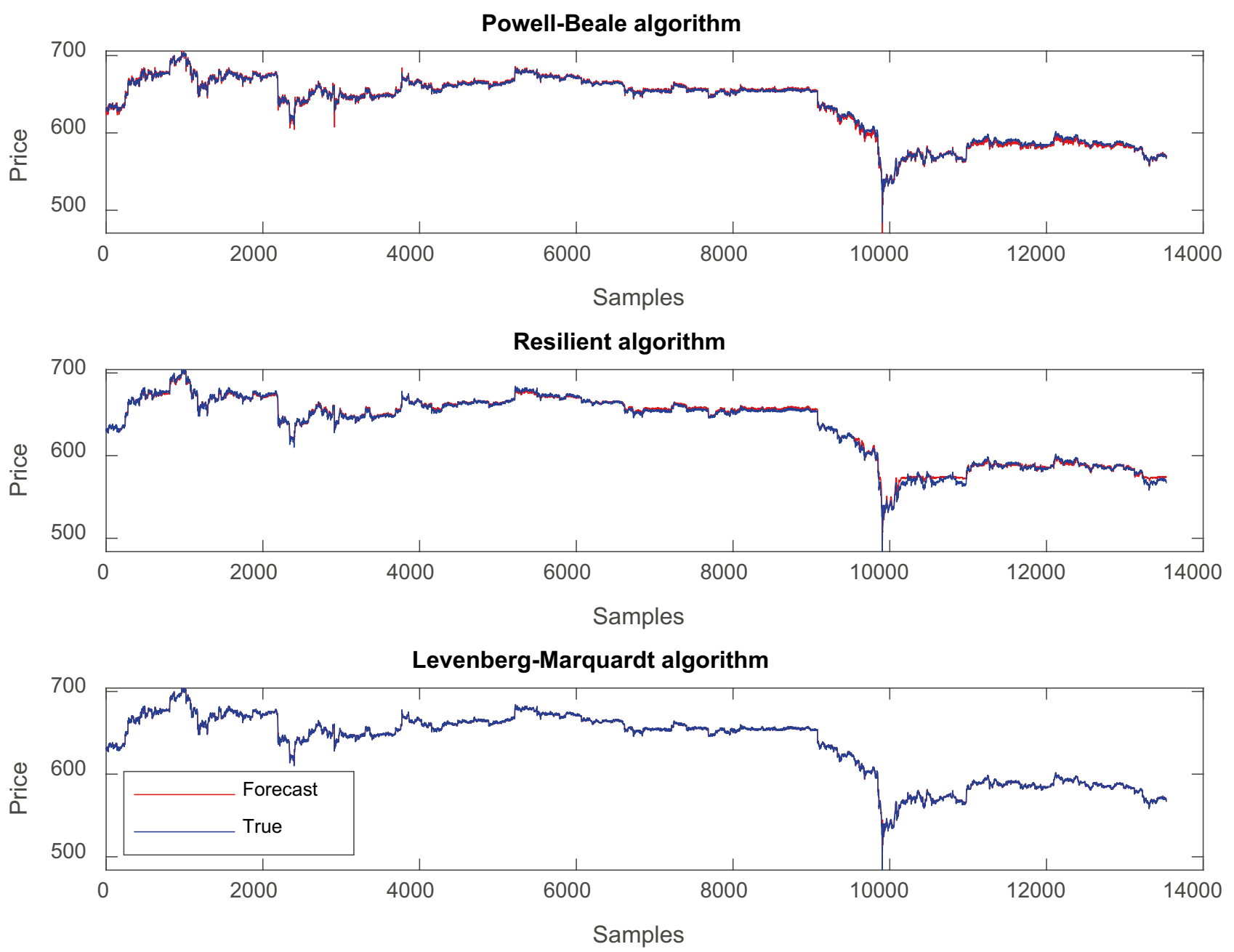

Fig. 1 Forecasted versus true Bitcoin price series by each DFFNN training algorithm

its forecasting performance as the choice of the training algorithm is determinant.

\section{Deep Feed-forward Neural Network Training}

The deep feed-forward neural network (DFFNN) [9] has several hidden layers. Specifically, the information in DFFNN moves from the input layer through the hidden layers to the output layer, and there is no feedback or loop in the network [9] which basically makes it deep and fast. A deep neural network architecture can learn very complex functions thanks to computations performed by multiple hidden layers to adjust its weights. The training of the DFFNN is performed by updating the change in its weights at each iteration according to three different numerical algorithms: conjugate gradient with Powell-Beale restarts, resilient algorithm, and Levenberg-Marquardt algorithm. The performance of the DFFNN under each training algorithm is evaluated by using the root mean of squared errors (RMSEs). The architecture of the DFFNN is organized as follows. The input layer has five neurons corresponding to the last observed prices of Bitcoin. There are three hidden layers, each with 20 neurons. Finally, the output layer has one single neuron corresponding to the forecasted Bitcoin price. Besides, the number of epochs is set to 50 , and the learning rate $\alpha$ is set to 0.001 .

\section{Data and Results}

The high frequency sampling of the Bitcoin intraday price data is at $5 \mathrm{~min}$ for the period from 1 January 2016 to 16 March 2018. Thus, the collected data has totally 65,535 observations. For simulation purposes, the first $80 \%$ samples of the full set are used for training each DFFNN and the remaining $20 \%$ are used for testing. Figure 1 shows the true and forecasted price series generated by each DFFNN 
on the testing sample. As shown, the DFFNN was able to generate forecasts close to the observed values under all three training algorithms. The comparison results show that the problem of Bitcoin high-frequency price time series prediction, the Levenberg-Marquardt algorithm performs the best $(\mathrm{RMSE}=1.4406)$, followed by PowellBeale restarts $(\mathrm{RMSE}=2.3187)$, and the resilient algorithm $(\mathrm{RMSE}=2.9715)$.

\section{Conclusions}

We evaluated a deep feed-forward neural network in highfrequency Bitcoin price time series forecasting under three different training algorithms including the conjugate gradient with Powell-Beale restarts, the resilient algorithm, and Levenberg-Marquardt algorithm. The experimental results demonstrated the superiority of the LevenbergMarquardt algorithm in terms of forecasting accuracy whilst the resilient algorithm performs the worst.

\section{Declarations}

Conflict of Interest The authors declare that they have no conflict of interest.

\section{References}

1. Fama EF. Random walks in stock market prices. Financial Anal J. 1995;51:75-80.

2. Bustos O, Pomares-Quimbaya A. Stock market movement forecast: a systematic review. Expert Syst Appl. 2020; 56, Article 113464.

3. Lahmiri S, Bekiros S. Cryptocurrency forecasting with deep learning chaotic neural networks. Chaos, Solitons Fractals. 2019;118:35-40.

4. Atsalakis GS, Atsalaki IG, Pasiouras F, Zopounidis C. Bitcoin price forecasting with neuro-fuzzy techniques. Eur J Oper Res. 2019;276:770-80.

5. Aggarwal D, Chandrasekaran S, Annamalai B. A complete empirical ensemble mode decomposition and support vector machine-based approach to predict Bitcoin prices. J Behav Exp Finance. 2020; 27, Article 100335.

6. Mallqui DCA, Fernandes RAS. Predicting the direction, maximum, minimum and closing prices of daily Bitcoin exchange rate using machine learning techniques. Appl Soft Comp. 2019;75:596-606.

7. Huang J-Z, Huang W, Ni J. Predicting bitcoin returns using high-dimensional technical indicators. J Finance Data Sci. 2019;5:140-55.

8. Lahmiri S, Bekiros S. Intelligent forecasting with machine learning trading systems in chaotic intraday Bitcoin market. Chaos, Solitons Fractals. 2020; 133, Article 109641.

9. Ketkar N. Feed Forward Neural Networks. Deep Learning with Python: Springer; 2017. p. 17-33. 